Modelling, Analysis and Simulation

Modelling, Analysis and Simulation

J. Wackers, B. Koren 
Centrum voor Wiskunde en Informatica (CWI) is the national research institute for Mathematics and Computer Science. It is sponsored by the Netherlands Organisation for Scientific Research (NWO).

CWI is a founding member of ERCIM, the European Research Consortium for Informatics and Mathematics.

CWI's research has a theme-oriented structure and is grouped into four clusters. Listed below are the names of the clusters and in parentheses their acronyms.

Probability, Networks and Algorithms (PNA)

Software Engineering (SEN)

\section{Modelling, Analysis and Simulation (MAS)}

Information Systems (INS)

Copyright (C) 2007, Stichting Centrum voor Wiskunde en Informatica

P.O. Box 94079, 1090 GB Amsterdam (NL)

Kruislaan 413, 1098 SJ Amsterdam (NL)

Telephone +31205929333

Telefax +31205924199

ISSN 1386-3703 


\title{
Efficient computation of steady water flow with waves
}

\author{
ABSTRACT \\ A surface capturing model for steady water flow is presented that can be solved very efficiently. \\ The model contains a high-accuracy water surface discretisation and turbulence; it is solved \\ with a novel linear multigrid technique and defect correction. Results show the accuracy of the \\ model and the fast convergence of the solver.
}

2000 Mathematics Subject Classification: 65N30;65N55;76F40

Keywords and Phrases: steady water waves; turbulence; volume-of-fluid; compressive limiter; multigrid; defect correction

Note: This research has been funded by the Dutch Bsik/BRICKS project in the theme MSV1.6. 



\title{
Efficient computation of steady water flow with waves
}

\author{
Jeroen Wackers ${ }^{1, *}$ and Barry Koren ${ }^{1,2}$ \\ ${ }^{1}$ CWI, P.O. Box 94079, 1090 GB Amsterdam, The Netherlands \\ 2 Delft University of Technology, Faculty of Aerospace Engineering, P.O. Box 5058, 2600 GB Delft, The Netherlands.
}

\begin{abstract}
SUMMARY
A surface capturing model for steady water flow is presented that can be solved very efficiently. The model contains a high-accuracy water surface discretisation and turbulence; it is solved with a novel linear multigrid technique and defect correction. Results show the accuracy of the model and the fast convergence of the solver.
\end{abstract}

KEY WORDS: steady water waves; turbulence; volume-of-fluid; compressive limiter; multigrid; defect correction

\section{INTRODUCTION}

Numerical simulation of steady water flow with gravity waves is of major importance in marine design. For example, accurate prediction of a ship's wave pattern and the interaction of the waves with the viscous flow near the ship hull enables the minimisation of the ship's drag in the design phase, reducing operating cost and environmental impact.

To model the water surface, two techniques exist: surface fitting and surface capturing [5]. For surface fitting, the grid is deformed during the computation, such that its upper boundary coincides with the water surface. This is a mature technique that gives accurate solutions and is computationally efficient. But it is not flexible: the grid deformation does not allow complex-shaped geometries.

For surface capturing, the grid is not deformed but the water surface moves through the grid. Examples are the volume-of-fluid and level set technique. These can handle arbitrary geometries and complex, even unstructured grids. The limiting factor for the use of surface capturing is the solution speed: as opposed to surface fitting, fast solution techniques are not readily available. Time stepping to convergence is the usual, costly solution technique.

Fast solution of surface capturing models is made difficult by the water surface model. To prevent excessive wave damping, an accurate model for the water surface is required. Usually, the location of the surface is reconstructed as a plane running through the grid and boundary conditions are imposed on that plane. It is hard to compute the flow field and this reconstructed plane simultaneously, so they

\footnotetext{
*Correspondence to: CWI, P.O. Box 94079, 1090 GB Amsterdam, The Netherlands. jeroen.wackers@ @wi.nl.
} 
must be updated alternately in a time stepping process. Furthermore, the high Reynolds numbers of water flow require a turbulence model in the flow equations; these usually need robust, slow solvers.

We show that a surface capturing model, with an accurate water surface discretisation and including a turbulence model, can be solved with the same efficiency as laminar flow models without a free surface. This is achieved with a volume-of-fluid ( $\mathrm{VoF}$ ) discretisation where the surface location is not reconstructed. The resulting flow model has no explicit surface model and consists of conservation laws only. Therefore, it is suitable for fast solvers.

We use a multigrid method to solve the steady flow equations, without any time stepping. Our novel multigrid technique is specially adapted for the solution of the VoF equation and the turbulence model. Second-order accuracy is obtained with defect correction; we present a compressive limiter for the second-order VoF fluxes that gives a highly accurate resolution of the water surface.

\section{FLOW EQUATIONS}

The flow is solved both in the water and in the air above it. The flow equations are based on the Reynolds-Averaged Navier-Stokes (RANS) equations; we distinguish between water and air by adding a mass conservation equation for the water. The water surface appears as a smeared out discontinuity, a smooth transition from water to air. In two dimensions, the system is:

$$
\begin{aligned}
\frac{\partial}{\partial x}\left(p+\rho u^{2}\right)+\frac{\partial}{\partial y}(\rho u v) & =\frac{\partial}{\partial x}\left(\left(\mu+\mu_{T}\right) 2 u_{x}\right)+\frac{\partial}{\partial y}\left(\left(\mu+\mu_{T}\right)\left(u_{y}+v_{x}\right)\right) & & (x \text {-mom. }), \\
\frac{\partial}{\partial x}(\rho u v)+\frac{\partial}{\partial y}\left(p+\rho v^{2}\right) & =\frac{\partial}{\partial x}\left(\left(\mu+\mu_{T}\right)\left(u_{y}+v_{x}\right)\right)+\frac{\partial}{\partial y}\left(\left(\mu+\mu_{T}\right) 2 v_{y}\right)-\rho g & & (y \text {-mom. }), \\
\frac{\partial}{\partial x}(u)+\frac{\partial}{\partial y}(v) & =0 & & \text { (tot. mass) }, \\
\frac{\partial}{\partial x}(u \alpha)+\frac{\partial}{\partial y}(v \alpha) & =0 & & \text { (water mass) },
\end{aligned}
$$

with $\alpha$ the volume fraction of water. The turbulent viscosity $\mu_{T}$ is computed with Menter's oneequation turbulence model [7]:

$$
\frac{\partial\left(\rho u v_{T}\right)}{\partial x}+\frac{\partial\left(\rho v v_{T}\right)}{\partial y}=\frac{\partial}{\partial x}\left(\left(\mu+\mu_{T}\right) \frac{\partial v_{T}}{\partial x}\right)+\frac{\partial}{\partial y}\left(\left(\mu+\mu_{T}\right) \frac{\partial v_{T}}{\partial y}\right)+P-D,
$$

with $v_{T}=\mu_{T} / \rho$. The production $P$ and the dissipation $D$ contain first and second velocity derivatives.

\section{FLUX DISCRETISATION}

The flow equations are discretised with cell-centred finite volumes on structured curvilinear grids. We use two different discretisations: multigrid is used to solve a first-order accurate discretisation and these solutions are improved with defect correction on a second-order accurate limited discretisation.

\subsection{Flux functions}

For robustness at high Reynolds numbers, the convective and diffusive fluxes are discretised separately. Stable convective fluxes are obtained with a Riemann solver. The states 0 and 1 on two sides of a cell 
face are used as input in a 1D shock tube problem; for this problem, the Navier-Stokes equations are made hyperbolic with artificial compressibility. The flux is set from the state between the waves in the shock tube solution. These convective fluxes are similar to those in [4], their derivation is given in [11]. The flux function couples the normal velocity $u$ and the pressure $p$, the output state $\frac{1}{2}$ is:

$$
u_{\frac{1}{2}}=u_{0}+\frac{p_{1}-p_{0}+\rho_{1} \lambda_{1}^{-}\left(u_{1}-u_{0}\right)}{\rho_{1} \lambda_{1}^{-}-\rho_{0} \lambda_{0}^{+}}, \quad p_{\frac{1}{2}}=p_{0}-\rho_{0} \lambda_{0}^{+} \frac{p_{1}-p_{0}+\rho_{1} \lambda_{1}^{-}\left(u_{1}-u_{0}\right)}{\rho_{1} \lambda_{1}^{-}-\rho_{0} \lambda_{0}^{+}},
$$

with the wave speeds defined as $\lambda^{-}=\frac{1}{2} u-\sqrt{c^{2} / \rho+(1 / 2 u)^{2}}$ and $\lambda^{+}=\frac{1}{2} u+\sqrt{c^{2} / \rho+(1 / 2 u)^{2}}$, for a constant $c$. The tangential velocity $v, v_{T}$, and $\alpha$ are chosen purely upwind, based on the sign of $u_{\frac{1}{2}}$.

The states on the two sides of a cell face are reconstructed from the cell centre states. For the firstorder accurate fluxes, the state at the cell faces is the state in the centre. The second-order scheme uses a Sweby-type limited reconstruction [8]. For example, at the left side of a vertical face the state is:

$$
q_{0}=q_{i, j}+\frac{1}{2} \phi\left(r_{0}\right)\left(q_{i, j}-q_{i-1, j}\right), \quad r_{0}=\frac{q_{i+1, j}-q_{i, j}}{q_{i, j}-q_{i-1, j}},
$$

We use the $\kappa=\frac{1}{3}$ limiter proposed by Koren [6], which is third-order accurate in one dimension:

$$
\phi_{\kappa}(r)=\max \left(0, \min \left(2 r, \frac{2}{3} r+\frac{1}{3}, 2\right)\right) .
$$

The diffusive fluxes and the turbulence source term are modelled with central differences. These are stable and second-order accurate, so they are used for both the first- and the second-order scheme.

\subsection{Compressive limiter for $\alpha$}

In our model, the volume fraction $\alpha$ is a smeared out discontinuity: $\alpha=1$ below the water surface, $\alpha=0$ in the air above, and the surface itself is a numerical mixing layer that contains both water and air. The solution is accurate when the surface is in the right location and when it is thin; the standard concept of first- and second-order accuracy has no meaning for a discontinuity.

Therefore, we can use a limiter for $\alpha$ that is not formally second-order accurate, like the limited downwind scheme:

$$
\phi_{L D}(r)=\max (0, \min (2 r, 2)) .
$$

This limiter follows the top of Sweby's monotonicity domain [8] so it is antidiffusive, it steepens any gradient into a discontinuity. Therefore, it keeps the surface sharp. However, the simple $\phi_{L D}$ limiter is too compressive: it deforms the interface into a staircase. The compressive limiters that are often used in time-dependent VoF methods [9] need to be very complex schemes, to prevent staircase deformation and make sure that $\alpha$ never becomes less than zero.

Our steady flow model allows us to use a simple compressive scheme. In steady flow, the interface is always parallel to the flow direction. Therefore, the interface location is fixed by the velocity field; large stairstep deformations cannot occur. And since the flow is in equilibrium, any monotone limiter guarantees that $\alpha$ stays between 0 and 1. In fact, the $\phi_{L D}$ limiter is satisfactory for steady flow. Still, following Ubbink and Issa [9], we use a hybrid limiter that switches to a second-order scheme when the interface is parallel to the cell face normal $\mathbf{n}$. This prevents even small stairsteps:

$$
\phi_{\alpha}=(1-\gamma) \phi_{L D}+\gamma \phi_{\kappa}, \quad \gamma=\frac{2}{\pi} \arccos \frac{|\mathbf{u} \cdot \mathbf{n}|}{\|\mathbf{u}\|} .
$$

Thus, the surface direction is given by the velocity field $\mathbf{u}$. The example in figure 1 shows that the compressive schemes give a constant surface width. For a small increase in thickness (3 cells instead of 2), the $\phi_{\alpha}$ limiter keeps the surface smooth, which improves the stability of the two-fluid flow model. 

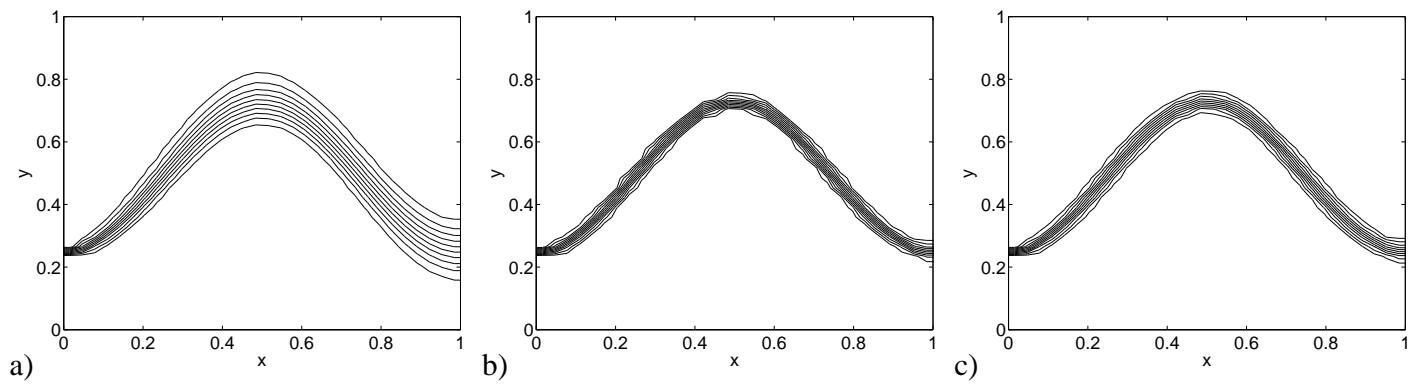

Figure 1. Solution of a linear test problem $(\mathbf{u} \cdot \nabla \alpha=0$ with $\mathbf{u}=[u, v]=[1,1.5 \sin 2 \pi x])$ on a $32 \times 32$ cell grid, for three different limiters: the second-order $\phi_{\kappa}$ (a) and the compressive $\phi_{L D}$ (b) and $\phi_{\alpha}$ (c).

\section{FAST SOLVER}

The heart of our fast solution method is a novel multigrid technique. The accuracy of the solutions is increased with defect correction.

\subsection{Improved line smoother}

Multigrid is combined with a powerful nonlinear collective line Gauss-Seidel smoother, that is effective for convection-dominated flow [11]. For pure convection-diffusion flows, the smoother always converges. However, the turbulence production term $P$ destabilises the system and may cause the smoothing to diverge. Therefore, most turbulent flow solvers permanently damp the smoothing.

We found that local damping in the first multigrid cycles is enough. Near a steady solution, the flow is stable, so undamped smoothing works once the solution process is partially converged. We damp the smoothing of $v_{T}$ in each individual line, when the Newton-Raphson solver that computes the update in that line does not converge. This guarantees the convergence of the line smoothing.

\subsection{Linear multigrid}

Standard nonlinear multigrid does not work for our system, because the flow equations on coarse grids differ too much from the fine grid equations. The water surface is a smeared discontinuity in $\alpha$, it is sharper on fine grids than on coarse grids. Also, the turbulence model does not model boundary layers accurately on coarse grids. Therefore, the coarse grid corrections in the multigrid algorithm become ineffective, as they rely on the similarity between the coarse and the fine grid equations.

Therefore, we use linear coarse grid corrections with Galerkin operators [10]. The fine grid flow equations are linearised (this linearisation is already computed for the line smoothing) and the linearisation is restricted directly to form the coarse grid equations. Thus, the coarse and fine grid equations are always similar. For robustness, the linear coarse grid correction is combined with nonlinear smoothing on the finest grid, that can remove unphysical solutions $\left(v_{T}<0\right.$ etc.).

\subsection{Defect correction}

Fast converging smoothers for the multigrid solution of second-order accurate equations are not available. Therefore, the second-order model is solved with defect correction. In each step, the residual of the second-order equations is put as a source term in the first-order equations, then a multigrid cycle 
is applied to the first-order equations. Defect correction converges slowly, but when it is started from the first-order accurate solution, a few steps are enough to make the solution second-order accurate [3].

For the two-fluid model, two changes are made to standard defect correction. First, the highly nonlinear flow equations cannot handle large source terms. Therefore, the second-order residual is scaled with a small constant; after each cycle, the change in state is unscaled, i.e. divided by the same constant. And second, near the water surface, the first- and second-order equations differ significantly. To keep the defect correction method stable, the corrections to the state there are limited such that $\alpha$ remains between 0 and 1 . In practice, this limiting is only needed in the air region.

\section{RESULTS}

Numerical results are shown for two 2D channel flows with a bottom bump, as measured by Cahouet [2]. Both are computed on $256 \times 256$ cell grids, with fine cells near the bottom and the free surface. The first test is a subcritical flow, with $F r=0.43$ (figure 2). For this flow, the grid is stretched at the inflow and outflow boundaries. For the first-order solution, the interface is smeared and the waves damp out quickly. The second-order solution after 8 defect correction steps shows a great improvement: the waves are higher and the interface is smeared less (it is about 4 cells thick). The compressive scheme makes the water surface, that smears at the inflow boundary, thinner near the first wave. Cahouet did not measure this case, a comparison with the numerical results of Van Brummelen [1] shows excellent agreement. A test with supercritical flow $(F r=2.05)$ is shown in figure 3 . Here, the first-order solution captures the shape of the wave, but the second-order solution ( 5 defect correction steps) is much sharper. The surface is $3-4$ cells thick throughout. The agreement with Cahouet is good.
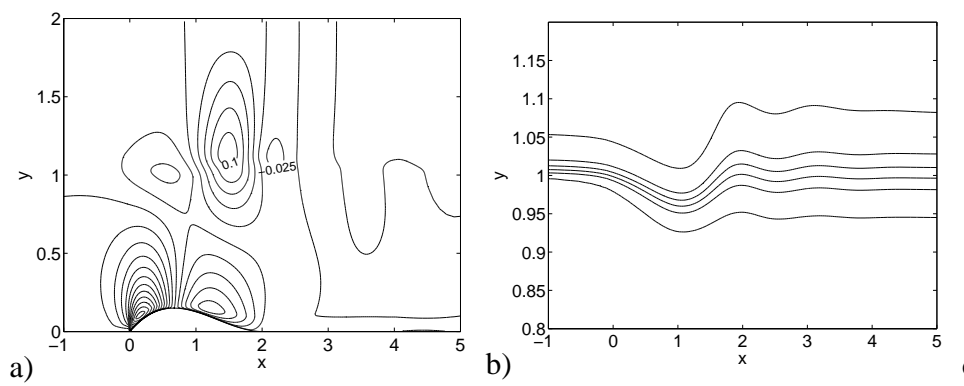

b)
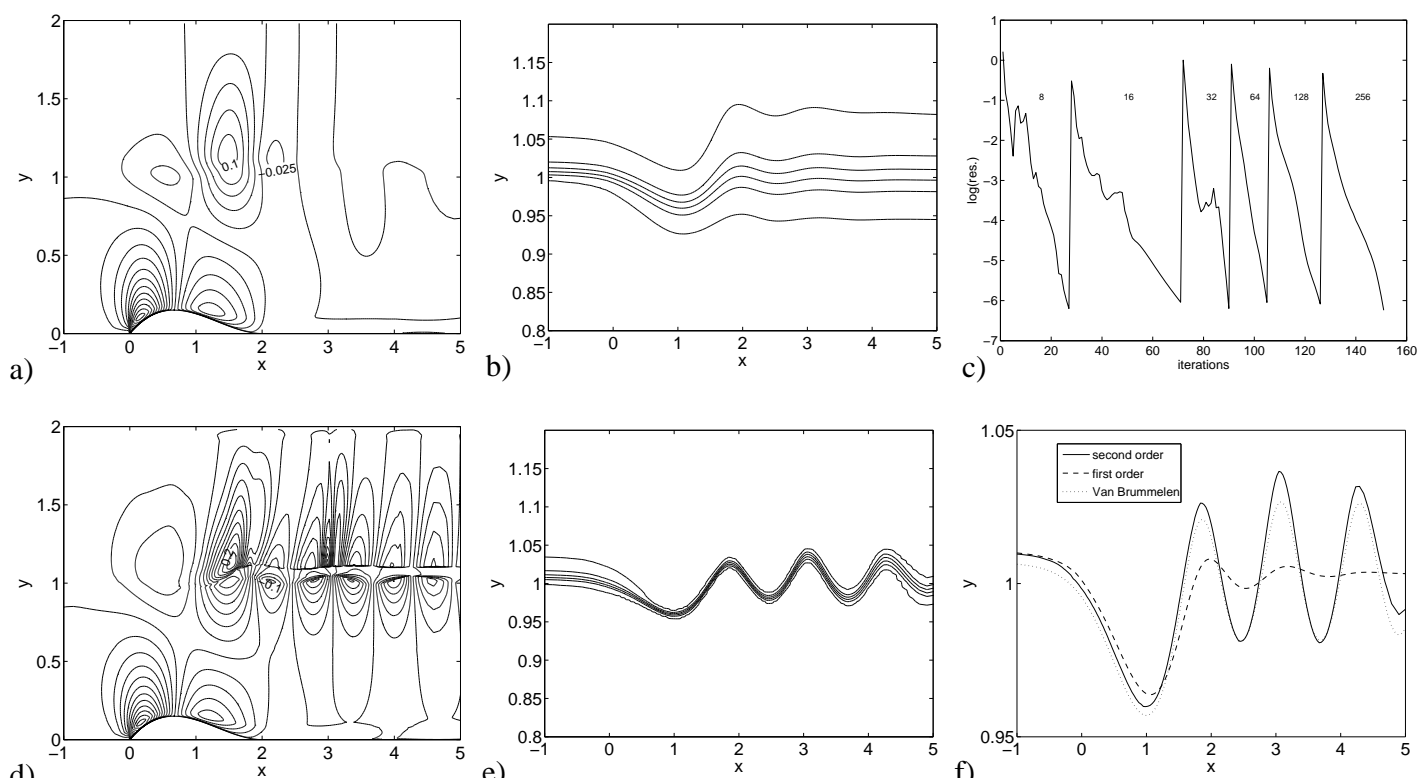

Figure 2. Solution of subcritical channel flow. First-order vertical velocity $v$ (a), volume fraction $\alpha$ (b), and the residual during the full multigrid computation on 8 grids (c). Second-order $v$ (c), $\alpha$ (d), and a comparison of both solutions with the numerical result of Van Brummelen [1] (e). Grid stretching starts at $x=4$. 

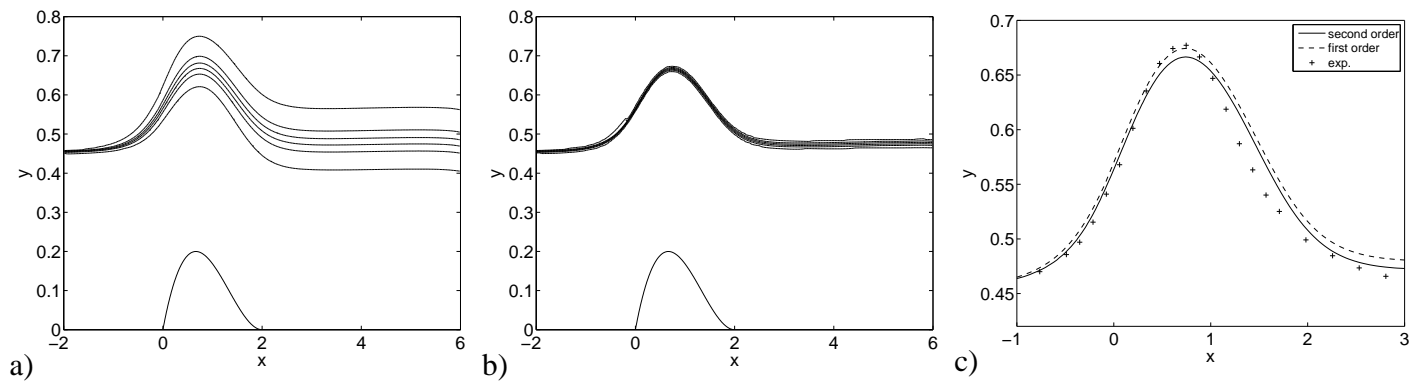

Figure 3. Supercritical channel flow test: the volume fraction for the first-order (a) and second-order accurate solution (b), and a comparison with Cahouet's experiment [2] (c).

The multigrid convergence for the first test case is shown in figure 2c. It is a full multigrid computation: the initial solution on each grid is found by propagating the solution on the next coarser grid. The convergence on the last grids is just as good as for the same problem with laminar flow [11]. The computation time is compared with a solution by line smoothing only: multigrid is about 20 times faster. Defect correction requires few cycles, it adds about a third to the first-order computation time.

\section{CONCLUSION}

A VoF model without interface reconstruction is proposed for steady water flow. The model consists of conservation laws only, so it can be solved with multigrid. Linear multigrid gives very fast convergence, even for turbulent flow. High-accuracy solutions and good agreement with experiments are obtained with a combination of defect correction and a compressive discretisation for the volume fraction.

\section{REFERENCES}

1. Van Brummelen EH. Numerical Methods for Steady Viscous Free-Surface Flows. PhD thesis, Universiteit van Amsterdam, Amsterdam, 2002.

2. Cahouet J. Etude numérique et expérimentale du problème bidimensionnel de la résistance de vagues non-linéaire. Technical report 185, Ecole Nationale Supérieure de Techniques Avancées: Paris, 1984.

3. Désidéri JA, Hemker PW. Convergence analysis of the defect-correction iteration for hyperbolic problems. SIAM Journal of Scientific Computing 1995; 16(1):88-118.

4. Dick E, Linden J. A multigrid method for steady incompressible Navier-Stokes equations based on flux difference splitting. International Journal for Numerical Methods in Fluids 1992; 14:1311-1323.

5. Hino T (ed). CFD Workshop Tokyo 2005. National Maritime Research Institute: Tokyo, 2005.

6. Koren B. A robust upwind discretization method for advection, diffusion and source terms. In Numerical Methods for Advection-Diffusion Problems, Vreugdenhil CB, Koren B (eds). Notes on Numerical Fluid Mechanics, volume 45. Vieweg: Braunschweig, 1993; 117-138.

7. Menter FR. Eddy viscosity transport equations and their relation to the $k-\varepsilon$ model. Journal of Fluids Engineering 1997; 119:876-884.

8. Sweby PK. High resolution schemes using flux limiters for hyperbolic conservation laws. SIAM Journal of Numerical Analysis 1984; 21:995-1011.

9. Ubbink O, Issa RI. A method for capturing sharp fluid interfaces on arbitrary meshes. Journal of Computational Physics $1999 ; 153: 26-50$.

10. Wackers J, Koren B. Accurate and efficient computation of steady water flow with surface waves and turbulence. In Proceedings of ECCOMAS CFD 2006, Wesseling P, Oñate E, Périaux J (eds). Delft University of Technology, 2006.

11. Wackers J, Koren B. A surface capturing method for the efficient computation of steady water waves. To appear in Journal of Computational and Applied Mathematics 2007 